\title{
Is it possible to construct exactly solvable models?
}

\author{
O. Haschke and W. Rühl \\ Department of Physics, University of Kaiserslautern, P.O.Box 3049 \\ 67653 Kaiserslautern, Germany
}

\begin{abstract}
We develop a constructive method to derive exactly solvable quantum mechanical models of rational (Calogero) and trigonometric (Sutherland) type. This method starts from a linear algebra problem: finding eigenvectors of triangular finite matrices. These eigenvectors are transcribed into eigenfunctions of a selfadjoint Schrödinger operator. We prove the feasibility of our method by constructing an " $A G_{3}$ model" of trigonometric type (the rational case was known before from Wolfes 1975). Applying a Coxeter group analysis we prove its equivalence with the $B_{3}$ model. In order to better understand features of our construction we exhibit the $F_{4}$ rational model with our method.
\end{abstract}

September 1998

Dedicated to Professor Jan Lopuszański on the occasion of his 75-th birthday 


\section{Introduction}

The completely integrable models are traditionally characterized by their relation with simple Lie algebras $A_{n}, B_{n}, C_{n}, D_{n}, G_{2}, F_{4}, E_{6}, E_{7}, E_{8}$. This relation is the starting point of the Hamiltonian reduction method exploited by Olshanetsky and Perelomov [1]. These models possess as limiting cases the trigonometric (Sutherland) and rational (Calogero) models that are exactly soluble, i.e. their eigenvalues and eigenvectors can be derived by elementary methods.

This exact solvability has been shown to follow from the fact that the Schrödinger operators can, after a "gauge transformation", be rewritten as a quadratic form of Lie algebra operators. These Lie algebra operators are represented as differential operators acting on polynomial spaces. This program was formulated in [2] and successfully applied first to the $A_{n}$ series in [3]. Then it was carried over to the other sequences $B_{n}, C_{n}, D_{n}$ and $G_{2}$ and even to corresponding supersymmetric models 4 , 5].

Our aim was to turn the arguments around and to develop an algorithm which may allow us to construct new exactly soluble models. First investigations were presented in [6]. The program contains two major and separate issues, to render a second order differential operator curvature free and to find a first order differential operator satisfying an integrability constraint. In this paper we present our algorithm in the following version. We start from a standard flat Laplacian and introduce Coxeter (or Weyl) group invariants as new coordinates. If the Coxeter group contains a symmetric group as subgroup, these invariants are built from elementary symmetric polynomials. The second order differential operators obtained this way are curvature free by construction, and act on polynomial spaces of these Coxeter invariants that form a flag. This flag is defined by means of a characteristic vector $(\vec{p}$-vector $)$.

Then we solve the integrability constraints by constructing "prepotentials" with a fixed algorithm. These prepotentials define the gauge transformation alluded to above which renders the differential operator the form of a standard Schrödinger operator of $N$ particles in 1-dimensional space with a potential. Each prepotential contributes an additive term to this potential with a free (real) coupling constant. Finally the prepotentials define the ground state wave function of the Schrödinger operator which originates from the trivial polynomial in the flag and thus contains no further information. Except a possible oscillator prepotential in the translation invariant cases, the prepotentials are in one-to-one relation with the orbits of the Coxeter group.

We show that all known exactly soluble models can be obtained this way (at present we have to make an exemption with respect to $E_{6}, E_{7}, E_{8}$, but this will soon be overcome). Applying the method of constructing the Coxeter 
invariants of $A_{2}$ [4] to $A_{3}$, we obtain an " $A G_{3}$ model". Its Coxeter diagram is that of the affine Coxeter group $\hat{B}_{3}$, which possesses the same invariants as the Coxeter group $B_{3}$. This leads to an explicit proof of the equivalence of the $A G_{3}$ model with the $B_{3}$ model. Thus a translation invariant four-particle model after separation of the c.m. motion is shown to be equivalent with a translation non-invariant three-particle model. In this paper we also discuss $F_{4}$ from the view point of our algorithm. The Schrödinger operator obtained (only the rational case) deviates slightly from the one given in [1] (probably due to a simple printing error in [四]).

Thus our method shifts the centre of interest from the simple Lie algebras and their homogeneous spaces to the corresponding Weyl groups and by generalization to the Coxeter groups. On the other hand, the differential operators acting on polynomial spaces of Coxeter invariants define Lie algebras of their own, but at present these algebras are only of marginal interest.

\section{The constructive program}

We are interested here in the bound state spectrum of Schrödinger operators. The whole analysis is therefore performed in real spaces. Consider a flag of polynomial spaces $V_{N}(\vec{p}), N \in \mathbb{Z}_{\geq}, \vec{p} \in \mathbb{N}^{n}$

$$
\begin{gathered}
V_{N}(\vec{p})=\operatorname{span}\left\{z_{1}^{r_{1}} z_{2}^{r_{2}} \ldots z_{n}^{r_{n}} \mid r_{1} p_{1}+r_{2} p_{2}+\ldots+r_{n} p_{n} \leq N\right\} \\
\left(p_{i} \in \mathbb{N}\right)
\end{gathered}
$$

We consider differential operators of first order

$$
D_{[\vec{\alpha} ; a]}^{(1)}=z^{[\vec{\alpha}]} \frac{\partial}{\partial z_{a}}
$$

( $\vec{\alpha}$ a multi-exponent)

and of second order

$$
D_{[\vec{\alpha} ; a, b]}^{(2)}=z^{[\vec{\alpha}]} \frac{\partial^{2}}{\partial z_{a} \partial z_{b}}
$$

that leave each space $V_{N}(\vec{p})$ invariant. If

$$
\vec{p}=(1,1, \ldots, 1)
$$

then the operators (2.2) generate the full linear (inhomogeneous) group of $\mathbb{R}_{n}$ and the operators of second order $(2.3)$ can be obtained as products from the first order operators, i.e. in (2.2)

$$
\vec{\alpha}=e^{(c)}, e_{b}^{(c)}=\delta_{b}^{c} \quad \text { or } \quad \vec{\alpha}=0
$$

and in $(2.3)$

$$
\vec{\alpha}=e^{(c)}+e^{(d)} \quad \text { or } \quad \vec{\alpha}=\mathrm{e}^{(\mathrm{c})} \quad \text { or } \quad \vec{\alpha}=0
$$


Now we consider a candidate for a future Schrödinger operator

$$
\begin{aligned}
D & =-\sum_{\vec{\alpha}, a, b} g_{[\vec{\alpha} ; a, b]} D_{[\vec{\alpha} ; a, b]}^{(2)} \\
& +\sum_{\vec{\beta}, c} h_{[\vec{\beta} ; c]} D_{[\vec{\beta} ; c]}^{(1)}
\end{aligned}
$$

The eigenvectors and values of $D$ in $V_{N}$ can be calculated easily by finite linear algebra methods. Let

$$
U_{N}=V_{N} / V_{N-1}
$$

and the diagonal part of $D$ on $U_{N}$ be defined as $D_{N}$

$$
D_{N} U_{N}=D U_{N} \cap U_{N}
$$

If the eigenvalues of $D_{N}$ are all different, the number of eigenvectors equals $\operatorname{dim} U_{N}$. But if some eigenvalues coincide (this is true in the generic case!) the number of eigenvectors is smaller. Then the Hilbert space on which the final selfadjoint Schrödinger operator is acting is not an $L^{2}$-space. The missing eigenfunctions can be described. For more details see [6].

If we want completely integrable models we must make sure that a complete set of involutive differential operators exists. For this task Lie algebraic methods may be very helpful.

Given a differential operator (2.7) one can characterize the vector $\vec{p}$ in (2.1) by inequalities

$$
\begin{aligned}
g_{[\vec{\alpha} ; a, b]} \neq 0 & \Rightarrow \vec{p} \vec{\alpha}-p_{a}-p_{b} \leq 0 \\
h_{[\vec{\beta} ; c]} \neq 0 & \Rightarrow \vec{p} \vec{\beta}-p_{c} \leq 0
\end{aligned}
$$

There should be enough equality signs in $(2.10),(2.11)$ for a chosen $\vec{p}$ so that $D_{N} \neq 0$. It turns out that there exists a minimal $\vec{p}$-vector $\vec{p}_{\min }$ so that the $V_{N}\left(\vec{p}_{\min }\right)$ spaces are maximal: For each $N, \vec{p}$ there is $N^{\prime}$ so that

$$
V_{N}(\vec{p}) \subset V_{N^{\prime}}\left(\vec{p}_{\min }\right)
$$

It is convenient to work only with this minimal $\vec{p}$-vector.

The first step in transforming $D$ into a Schrödinger operator is to write it symmetrically

$$
D=-\sum_{a, b} \frac{\partial}{\partial z_{a}} g_{a b}^{-1}(z) \frac{\partial}{\partial z_{b}}+\sum_{a} r_{a}(z) \frac{\partial}{\partial z_{a}}
$$

where

$$
g_{a b}^{-1}=\sum_{\vec{\alpha}} g_{[\vec{\alpha} ; a, b]} z^{[\vec{\alpha}]}
$$


We write $g_{a b}^{-1}$ because this is the inverse of a Riemann tensor. The Riemann tensor $g_{a b}$ is assumed to be curvature free. The task to make it so will not arise in this work. But we mention that we developed a minimal algorithm to solve this issue.

Following the notations of [6] we "gauge" the polynomial eigenfunctions $\varphi$ of $D$ by

$$
\psi(z)=e^{-\chi(z)} \varphi(z)
$$

so that

$$
e^{-\chi} D e^{+\chi}=-\frac{1}{\sqrt{g}} \sum_{a, b} \frac{\partial}{\partial z_{a}}\left(\sqrt{g} g_{a b}^{-1}\right) \frac{\partial}{\partial z_{b}}+W(z)
$$

$\left(g=\left(\operatorname{det} g^{-1}\right)^{-1}\right)$.

This is possible if and only if

$$
\sum_{b} g_{a b}^{-1}(z) \frac{\partial}{\partial z_{b}}[2 \chi-\ln \sqrt{g}]=r_{a}(z)
$$

which implies integrability constraints on the functions $\left\{r_{a}(z)\right\}$. If they are fulfilled we obtain a "prepotential"

$$
\rho=\ln P
$$

so that

$$
\rho=2 \chi-\ln \sqrt{g}
$$

In most cases studied, we found solutions for $\rho$ as follows. Let

$$
\operatorname{det} g^{-1}(z)=\prod_{i=1}^{r} P_{i}(z)
$$

where $\left\{P_{i}(z)\right\}$ are different real polynomials. Then

$$
\rho(z)=\sum_{i=1}^{r} \gamma_{i} \ln P_{i}(z)
$$

with free parameters $\gamma_{i}$ solves the requirement that $\left\{r_{a}(z)\right\}$ (2.17) belong to differential operators leaving each $V_{N}$ invariant. In particular

$$
r_{a}^{(i)}(z)=\frac{1}{P_{i}(z)} \sum_{b} g_{a b}^{-1}(z) \frac{\partial P_{i}}{\partial z_{b}}
$$

are polynomials. Inserting $(2.20),(2.21)$ in $(2.19)$ we obtain finally

$$
\chi=\frac{1}{2} \sum_{i=1}^{r}\left(\gamma_{i}-\frac{1}{2}\right) \ln P_{i}
$$


We will later see that in the case of the models of Calogero type a term

$$
\gamma_{0} \ln P_{0}
$$

can be added to $\rho$, where

$$
P_{0}(z)=e^{z_{1}}
$$

is not contained in $\operatorname{det} g^{-1}$ as a factor. This prepotential gives rise to the oscillator potential.

Finally we mention that $e^{-\chi}$ is the ground state wave function of the Schrödinger operator, as follows from (2.15).

The expression [6], (6.17) for the potential $W(z)$ contains a term linear in $\chi$

$$
-\sum_{a, b} \frac{\partial}{\partial z_{a}}\left(g_{a b}^{-1} \frac{\partial \chi}{\partial z_{b}}\right)=-\frac{1}{2} \sum_{i=1}^{r}\left(\gamma_{i}-\frac{1}{2}\right) \sum_{a} \frac{\partial}{\partial z_{a}} r_{a}^{(i)}
$$

Each divergence

$$
\sum_{a} \frac{\partial}{\partial z_{a}} r_{a}^{(i)}(z)=C^{(i)}
$$

ought to be a constant. From now on we shall dismiss all constant terms in $W(z)$.

We can then write the potential as

$$
\begin{gathered}
W(z)=\sum_{i, j} \gamma_{i j} R_{i j}(z) \\
R_{i j}=\sum_{a, b} g_{a b}^{-1} \frac{\partial \ln P_{i}}{\partial z_{a}} \frac{\partial \ln P_{j}}{\partial z_{b}} \\
\gamma_{i j}=\frac{1}{4}\left(\gamma_{i} \gamma_{j}-\frac{1}{4}\right) \quad(i, j \neq 0) .
\end{gathered}
$$

In the cases of this article

$$
R_{i j}=\text { const if } i \neq j
$$

If we then set

$$
\gamma_{i}=-\nu_{i}+\frac{1}{2} \quad(i \neq 0)
$$

we obtain

$$
W(z)=\sum_{i=1}^{r} \gamma_{i i} R_{i i}(z)
$$

with

$$
\gamma_{i i}=\frac{1}{4} \nu_{i}\left(\nu_{i}-1\right)
$$

As stated in the Introduction the variables $\left\{z_{i}\right\}$ appearing in this section are identified with Coxeter invariants formed from root space coordinates 
$\left\{x_{n}\right\}$ or $\left\{y_{n}\right\}$. These invariants are either polynomial or trigonometric. Finally we return from the invariant coordinates $\left\{z_{i}\right\}$ to the root space coordinates $\left\{x_{n}\right\}$ in the Schrödinger operator (2.16). Each contribution

$$
R_{i i}=\frac{Q_{i i}}{P_{i}}
$$

admits a partial fraction decomposition due to the factorization of the prepotentials $P_{i}$ (Section 5). The label $i=1$ is always reserved to a "Vandermonde prepotential", i.e.

$$
P_{1} \sim \prod_{i<j}\left(x_{i}-x_{j}\right)^{2} \quad \text { or } \quad \prod_{i<j}\left(\sin \left(x_{i}-x_{j}\right)\right)^{2}
$$

or alike.

\section{Translation invariant models}

\subsection{Relative coordinates}

The Laplacian for an Euclidean space $\mathbb{R}_{N}$

$$
\Delta=\sum_{i=1}^{N} \frac{\partial^{2}}{\partial x_{i}^{2}}
$$

is translation invariant. We introduce relative coordinates by

$$
\begin{aligned}
& y_{i}=x_{i}-\frac{1}{N} X \\
& X=\sum_{i=1}^{N} x_{i}
\end{aligned}
$$

They separate the Laplacian such that

$$
\Delta=N \frac{\partial^{2}}{\partial X^{2}}+\sum_{i=1}^{N} \frac{\partial^{2}}{\partial y_{i}^{2}}-\frac{1}{N}\left(\sum_{i=1}^{N} \frac{\partial}{\partial y_{i}}\right)^{2}
$$

We use all $\left\{y_{i}\right\}_{i=1}^{N}$ as coordinates on the plane

$$
\sum_{i=1}^{N} y_{i}=0
$$

in order to maintain permutation symmetry. 


\subsection{Elementary symmetric polynomials}

Elementary symmetric polynomials of $N$ variables $\left\{q_{i}\right\}_{i=1}^{N}$ are defined by a generating function

$$
\sum_{n=0}^{N} p_{n}(q) t^{n}=\prod_{i=1}^{N}\left(1+q_{i} t\right)
$$

They are invariant under the symmetric group $S_{N}$. For each $g \in S_{N}$ we have a sector (simplex) $E_{g} \subset \mathbb{R}_{N}$

$$
E_{g}=\left\{q_{i_{1}}<q_{i_{2}}<\ldots<q_{i_{N}} ; \quad i_{n}=g(n)\right\}
$$

so that

$$
\mathbb{R}_{N}=\bigcup_{g \in S_{N}} \bar{E}_{g}
$$

Inside $E_{g}$ we can use the $\left\{p_{n}\right\}_{n=1}^{N}$ as coordinates since

$$
\begin{gathered}
\mathcal{M}_{n i}=\frac{\partial p_{n}}{\partial q_{i}} \\
\operatorname{det} \mathcal{M}=(-1)^{\left[\frac{N}{2}\right]} V\left(q_{1}, q_{2}, \ldots q_{N}\right)
\end{gathered}
$$

where $V$ is the Vandermonde determinant.

\subsection{The $A_{N-1}$ series}

The root system of $A_{N-1}$ and the corresponding Weyl group possess elementary symmetric polynomials as invariants. We express the Laplacian in each sector $E_{g}(3.7)$ intersected with the plane (3.5) in terms of these polynomials

$$
\tau_{n}\left(y_{1}, \ldots, y_{N}\right)=\left.p_{n}(q)\right|_{q_{i}=y_{i} \text { all } i}
$$

The dynamics will be bounded to such sectors by corresponding potential walls automatically.

Then (see [3]) it results

$$
\begin{array}{r}
\sum_{i=1}^{N} \frac{\partial^{2}}{\partial y_{i}^{2}}-\frac{1}{N}\left(\sum_{i=1}^{N} \frac{\partial}{\partial y_{i}}\right)^{2} \\
=\sum_{n, m=2}^{N} g_{n m}^{-1} \frac{\partial^{2}}{\partial \tau_{n} \partial \tau_{m}}+\sum_{n=2}^{N} h_{n} \frac{\partial}{\partial \tau_{n}}
\end{array}
$$

with

$$
g_{n m}^{-1}(\tau)=\frac{1}{N}(m-1)(N-n+1) \tau_{n} \tau_{m}-T_{n-1, m-1}(\tau)
$$

and

$$
T_{n m}(\tau)=\sum_{l \geq 1}(2 l+n-m) \tau_{n+l} \tau_{m-l}
$$


Here it is understood that

$$
\begin{aligned}
\tau_{0} & =1 \\
\tau_{1} & =0 \\
\tau_{n} & =0 \text { for } n<0, n>N
\end{aligned}
$$

In this case $\operatorname{det} g^{-1}$ is indecomposable as a polynomial, so we set

$$
\begin{aligned}
& P_{0}=e^{\omega \tau_{2}} \\
& P_{1}=\operatorname{det} g^{-1}=C_{N} V\left(y_{1}, \ldots, y_{N}\right)^{2}
\end{aligned}
$$

The resulting vectors $\left\{r_{a}\right\}_{2}^{N}$ are

$$
\begin{gathered}
r^{(0)}=\left(-2 \tau_{2},-3 \tau_{3}, \ldots,-N \tau_{N}\right) \\
r^{(1)}: \text { explicit formulas known only for } N \leq 4
\end{gathered}
$$

and the potential is

$$
\frac{1}{2} W(x)=\frac{1}{2} \omega^{2} \sum_{i=1}^{N} x_{i}^{2}+g \sum_{1 \leq i<j \leq N}\left(x_{i}-x_{j}\right)^{-2}
$$

The corresponding Sutherland models are obtained as follows. We use as coordinates a system $\left\{\sigma_{n}\right\}_{n=2}^{N}$ defined by (these differ from those in [3])

$$
\sigma_{0}=\prod_{i=1}^{N} \cos y_{i}
$$

and

$$
\sigma_{n}=\left.\sigma_{0} \cdot p_{n}(q)\right|_{q_{i}=\tan y_{i}}
$$

The identity

$$
\begin{aligned}
1 & =\exp \left(i \sum_{j=1}^{N} y_{j}\right) \\
& =\prod_{j=1}^{N}\left(\cos y_{j}+i \sin y_{j}\right) \\
& =\sum_{n=0}^{N} i^{n} \sigma_{n}(y)
\end{aligned}
$$

allows us to eliminate $\sigma_{0}$ and $\sigma_{1}$ in terms of the remaining $\left\{\sigma_{n}\right\}_{n=2}^{N}$ so that polynomials go into polynomials. 
The Laplacian is expressed correspondingly as

$$
\begin{aligned}
& \sum_{i=1}^{N} \frac{\partial^{2}}{\partial y_{1}^{2}}-\frac{1}{N}\left(\sum_{i=1}^{N} \frac{\partial}{\partial y_{i}}\right)^{2}= \\
= & \sum_{n, m=2}^{N} g_{n m}^{-1} \frac{\partial^{2}}{\partial \sigma_{n} \partial \sigma_{m}}+\sum_{n=2}^{N} h_{n} \frac{\partial}{\partial \sigma_{n}} \\
g_{n m}^{-1}(\sigma)= & -T_{n+1, m+1}(\sigma)-T_{n+1, m-1}(\sigma) \\
& -T_{n-1, m+1}(\sigma)-T_{n-1, m-1}(\sigma) \\
& +\frac{1}{N}\left[(m+1) \sigma_{m+1}+(m-1) \sigma_{m-1}\right] \\
& \times\left[(N-n-1) \sigma_{n+1}+(N-n+1) \sigma_{n-1}\right]
\end{aligned}
$$

with $T_{n m}$ as in (3.14).

Once again $\operatorname{det} g^{-1}$ is indecomposable, so we set

$$
P_{1}=\operatorname{det} g^{-1}=C_{N}^{\prime} \tilde{V}\left(y_{1}, \ldots, y_{N}\right)^{2}
$$

where

$$
\tilde{V}\left(y_{1}, \ldots, y_{N}\right)=\prod_{i<j} \sin \left(y_{i}-y_{j}\right)
$$

has the symmetry of the Vandermonde determinant (translations and permutations). The vector $r^{(1)}$ is known only up to $N=4$. Finally we obtain as potential

$$
\frac{1}{2} W(x)=g \sum_{1 \leq i<j \leq N} \sin \left(x_{i}-x_{j}\right)^{-2}
$$

In each case $A_{N-1}$ the minimal $p$-vector is $(1,1, \ldots, 1) \in \mathbb{N}^{N-1}$.

\subsection{The $G_{2}$ and $A G_{3}$ models}

The models $G_{2}$ and $A G_{3}$ belong also to the domain of translation invariant models [4]. For $G_{2}$ we start from $A_{2}$ and extend its Weyl group by a $\mathbb{Z}_{2}$ group

$$
y_{i} \rightarrow-y_{i}
$$

As invariant variables we use [䧃]

$$
\begin{aligned}
& \lambda_{2}=\tau_{2} \\
& \lambda_{3}=\tau_{3}^{2}
\end{aligned}
$$


In these variables

$$
\begin{array}{r}
\sum_{i=1}^{3} \frac{\partial^{2}}{\partial y_{i}^{2}}-\frac{1}{3}\left(\sum_{i=1}^{3} \frac{\partial}{\partial y_{i}}\right)^{2}= \\
=\sum_{a, b=2}^{3} g_{a b}^{-1} \frac{\partial^{2}}{\partial \lambda_{a} \partial \lambda_{b}}+\sum_{a=2}^{3} h_{a} \frac{\partial}{\partial \lambda_{a}}
\end{array}
$$

We find

$$
g^{-1}(\lambda)=\left(\begin{array}{cc}
-2 \lambda_{2}, & -6 \lambda_{3} \\
-6 \lambda_{3}, & +\frac{8}{3} \lambda_{2}^{2} \lambda_{3}
\end{array}\right)
$$

so that

$$
\operatorname{det} g^{-1}=-\frac{4}{3} \lambda_{3}\left(4 \lambda_{2}^{3}+27 \lambda_{3}\right)
$$

Thus as ansatz for the prepotentials we use

$$
\begin{aligned}
& P_{0}=e^{\omega \lambda_{2}} \\
& P_{1}=4 \lambda_{2}^{3}+27 \lambda_{3} \\
& P_{2}=\lambda_{3}
\end{aligned}
$$

The $r$-vectors (justifying this ansatz) are

$$
\begin{aligned}
r^{(0)} & =\left(-2 \lambda_{2},-6 \lambda_{3}\right) \\
r^{(1)} & =(-6,0) \\
r^{(2)} & =\left(-6,+\frac{8}{3} \lambda_{2}^{2}\right)
\end{aligned}
$$

The minimal $\vec{p}$-vector is

$$
\vec{p}=(1,2)
$$

The potential is

$$
\begin{aligned}
\frac{1}{2} W(x)= & \frac{1}{2} \omega^{2} \sum_{i=1}^{3} x_{i}^{2} \\
& +g_{1} \sum_{1 \leq i<j \leq 3}\left(x_{i}-x_{j}\right)^{-2}+g_{2} \sum_{i<j, k \notin(i, j)}\left(x_{i}+x_{j}-2 x_{k}\right)^{-2}
\end{aligned}
$$

with

$$
\begin{aligned}
& g_{1}=\nu_{1}\left(\nu_{1}-1\right) \\
& g_{2}=3 \nu_{2}\left(\nu_{2}-1\right)
\end{aligned}
$$

If

$$
\nu_{2}=0 \text { or } \nu_{2}=1
$$

we return to the $A_{2}$ model. 
In the Sutherland case we use as variables

$$
\begin{aligned}
& \mu_{2}=\sigma_{2} \\
& \mu_{3}=\sigma_{3}^{2}
\end{aligned}
$$

leading to the inverse Riemann tensor

$$
g^{-1}=\left(\begin{array}{ll}
-2 \mu_{2}-2 \mu_{2}^{2}+\frac{2}{3} \mu_{3}, & -\mu_{3}\left(6+\frac{16}{3} \mu_{2}\right) \\
-\mu_{3}\left(6+\frac{16}{3} \mu_{2}\right), & \frac{8}{3} \mu_{2}^{2} \mu_{3}-8 \mu_{3}^{2}
\end{array}\right)
$$

Now det $g^{-1}$ is decomposable with

$$
\operatorname{det} g^{-1}=-\frac{4}{3} \mu_{3} P_{1}(\mu)
$$

and

$$
\begin{gathered}
P_{1}(\mu)=4 \mu_{3}^{2}+\mu_{3}\left(8 \mu_{2}^{2}+36 \mu_{2}+27\right)+4 \mu_{2}^{3}\left(1+\mu_{2}\right) \\
P_{2}(\mu)=\mu_{3}
\end{gathered}
$$

The $r$-vectors are

$$
\begin{aligned}
r^{(1)} & =\left(-6-8 \mu_{2},-16 \mu_{3}\right) \\
r^{(2)} & =\left(-6-\frac{16}{3} \mu_{2}, \frac{8}{3} \mu_{2}^{2}-16 \mu_{3}\right)
\end{aligned}
$$

The resulting potential is

$$
\begin{aligned}
\frac{1}{2} W(x)= & g_{1} \sum_{1 \leq i<j \leq 3} \sin \left(x_{i}-x_{j}\right)^{-2} \\
& +\frac{1}{9} g_{2} \sum_{i<j, k \notin(i, j)} \sin \frac{1}{3}\left(x_{i}+x_{j}-2 x_{k}\right)^{-2}
\end{aligned}
$$

In the case of the $A_{2}$ models the spaces $V_{N}$ decompose into even and odd subspaces in $\tau_{3}$ (or $\sigma_{3}$ ) which are left invariant separately under action of the Laplacian. In the case of the odd spaces we can factor $\tau_{3}\left(\sigma_{3}\right)$ and leave an even space as well. In each case we obtain a polynomial space in the variables $\lambda_{2}, \lambda_{3}=\tau_{3}^{2}\left(\mu_{2}, \mu_{3}=\sigma_{3}^{2}\right)$. Thus starting from such polynomial space and multiplying with $\tau_{3}^{\nu_{2}}\left(\sigma_{3}^{\nu_{2}}\right)$ we obtain the $A_{2}$ model if $\nu_{2}=0$ or $\nu_{2}=1$ but a new potential in all other cases.

It is plausible that a similar procedure works for $A_{3}$ but not for $A_{N-1}, N \geq$ 5 . In the latter models we have two or more odd variables $\tau_{3}, \tau_{5}, \ldots\left(\sigma_{3}, \sigma_{5}, \ldots\right)$ and there is no factorization of the odd invariant subspaces. Let us sketch the $A_{3}$ model whose extension leads to the $A G_{3}$ model [8].

In this case the variables are chosen as in (3.29), (3.30), (3.44), (3.45)

$$
\lambda_{2}=\tau_{2}, \lambda_{3}=\tau_{3}^{2}, \lambda_{4}=\tau_{4}
$$


The inverse Riemann tensor is

$$
g^{-1}=\left(\begin{array}{ccc}
-2 \lambda_{2}, & -6 \lambda_{3}, & -4 \lambda_{4} \\
-6 \lambda_{3}, & 4 \lambda_{3}\left(\lambda_{2}^{2}-4 \lambda_{4}\right), & \lambda_{2} \lambda_{3} \\
-4 \lambda_{4}, & +\lambda_{2} \lambda_{3}, & -2 \lambda_{2} \lambda_{4}+\frac{3}{4} \lambda_{3}
\end{array}\right)
$$

The determinant is decomposable as

$$
\operatorname{det} g^{-1}=\lambda_{3} P_{1}(\lambda)
$$

and the ansatz for the prepotentials is

$$
\begin{aligned}
P_{0}(\lambda)= & e^{\omega \lambda_{2}} \\
P_{1}(\lambda)= & 27 \lambda_{3}^{2}-256 \lambda_{4}^{3}+128 \lambda_{2}^{2} \lambda_{4}^{2} \\
& -16 \lambda_{2}^{4} \lambda_{4}+4 \lambda_{2}^{3} \lambda_{3}-144 \lambda_{2} \lambda_{3} \lambda_{4} \\
P_{2}(\lambda)= & \lambda_{3}
\end{aligned}
$$

The $r$-vectors come out as

$$
\begin{aligned}
& r^{(0)}=\left(-2 \lambda_{2},-6 \lambda_{3},-4 \lambda_{4}\right) \\
& r^{(1)}=\left(-12,0,-2 \lambda_{2}\right) \\
& r^{(2)}=\left(-6,4\left(\lambda_{2}^{2}-4 \lambda_{4}\right), \lambda_{2}\right)
\end{aligned}
$$

The potential for this Calogero type model is

$$
\begin{aligned}
\frac{1}{2} W(x)= & \frac{1}{2} \omega^{2} \sum_{i=1}^{4} x_{i}^{2} \\
& +g_{1} \sum_{1 \leq i<j \leq 4}\left(x_{i}-x_{j}\right)^{-2}+g_{2} \sum_{3 \text { terms }}\left(x_{i}+x_{j}-x_{k}-x_{l}\right)^{-2}
\end{aligned}
$$

with

$$
g_{1}=\nu_{1}\left(\nu_{1}-1\right), g_{2}=2 \nu_{2}\left(\nu_{2}-1\right)
$$

It was discovered first by Wolfes, [7].

The Sutherland model is obtained in the same fashion. With

$$
\mu_{2}=\sigma_{2}, \mu_{3}=\sigma_{3}^{2}, \mu_{4}=\sigma_{4}
$$

the inverse Riemann tensor is

$$
\begin{aligned}
& g_{22}^{-1}=-2 \mu_{2}-2 \mu_{2}^{2}-8 \mu_{4}+2 \mu_{3}+8 \mu_{2} \mu_{4}+8 \mu_{4}^{2} \\
& g_{23}^{-1}=-6 \mu_{3}-4 \mu_{2} \mu_{3} \\
& g_{24}^{-1}=-4 \mu_{4}-6 \mu_{2} \mu_{4}+\mu_{3}+4 \mu_{4}^{2} \\
& g_{33}^{-1}=4 \mu_{3}\left[-4 \mu_{4}+\mu_{2}^{2}-4 \mu_{2} \mu_{4}+4 \mu_{4}^{2}-2 \mu_{3}\right] \\
& g_{34}^{-1}=\mu_{2} \mu_{3}-6 \mu_{3} \mu_{4} \\
& g_{44}^{-1}=-2 \mu_{2} \mu_{4}+\frac{3}{4} \mu_{3}
\end{aligned}
$$


Its determinant decomposes

$$
\begin{gathered}
\operatorname{det} g^{-1}=-\mu_{3} P_{1}(\mu) \\
P_{1}(\mu)=256 \mu_{4}^{6}+32 \text { further terms } \\
\text { (equ. (A.2) from [8]) } \\
P_{2}(\mu)=\mu_{3}
\end{gathered}
$$

and the $r$-vectors are

$$
\begin{gathered}
r^{(1)}=\left(-16 \mu_{2}-12,-24 \mu_{3},-12 \mu_{4}-2 \mu_{2}\right) \\
r^{(2)}=\left(-4 \mu_{2}-8,16 \mu_{4}^{2}-16 \mu_{4} \mu_{2}+4 \mu_{2}^{2}-8 \mu_{3}-16 \mu_{4},-6 \mu_{4}+\mu_{2}\right)
\end{gathered}
$$

The factorization of $\sigma_{3}$ which is necessary in this case is

$$
\sigma_{3}=-\prod_{1 \leq i<j \leq 3} \sin \left(y_{i}+y_{j}\right)
$$

implying

$$
\frac{Q_{22}}{P_{2}}=4 \sum_{1 \leq i<j \leq 3}\left(\sin \left(y_{i}+y_{j}\right)\right)^{-2}
$$

This gives the potential

$$
\begin{aligned}
\frac{1}{2} W(x)= & g_{1} \sum_{1 \leq i<j \leq 4}\left(\sin \left(x_{i}-x_{j}\right)\right)^{-2} \\
& +\frac{1}{4} g_{2} \sum_{3 \text { cases }}\left(\sin \frac{1}{2}\left(x_{i}+x_{j}-x_{k}-x_{l}\right)\right)^{-2}
\end{aligned}
$$

The discussion of this $A G_{3}$ model is resumed in Section 5.

\section{Translation non-invariant models}

\subsection{The $B C_{N}$ and $D_{N}$ models}

As we shall see there is only one series with two (Calogero) and three (Sutherland) independent coupling constants. For any such model we use as Cartesian coordinates $\left\{x_{i}\right\}_{i=1}^{N}$ and require permutation symmetry $S_{N}$ and reflection symmetry $\left(\mathbb{Z}_{2}\right)^{N} x_{i} \rightarrow-x_{i}$ for each $i$ separately. Then the natural coordinates invariant under these group actions are [5]

$$
\lambda_{n}(x)=\left.p_{n}(q)\right|_{q_{i}=x_{i}^{2}, \text { all } i}
$$

There is a bilinear relation with the $\left\{p_{n}(x)\right\}_{n=1}^{N}$

$$
\lambda_{n}(x)=\sum_{k=0}^{2 n}(-1)^{n-k} p_{2 n-k}(x) p_{k}(x)
$$


The inverse Riemann tensor for the full Laplacian (3.1) is then

$$
g_{n m}^{-1}(\lambda)=4 M_{n m}(\lambda)
$$

where we introduce the shorthand

$$
M_{n m}(\lambda)=\sum_{l \geq 0}(2 l+n-m+1) \lambda_{n+l} \lambda_{m-1-l}
$$

Its determinant factorizes

$$
\operatorname{det} g^{-1}=(-1)^{\left[\frac{N}{2}\right]} 4^{N} \lambda_{N} P_{1}(\lambda)
$$

where

$$
\begin{aligned}
P_{1}(\lambda) & =N^{N} \lambda_{N}^{N-1}+\ldots \\
& =D_{N} V\left(x_{1}^{2}, x_{2}^{2}, \ldots x_{N}^{2}\right)^{2}
\end{aligned}
$$

and

$$
P_{2}(\lambda)=\lambda_{N}
$$

Both functions $P_{1}, P_{2}$ factorize in a trivial way. In the general case there is no explicit expression for $r^{(1)}$ but

$$
r_{a}^{(2)}=4(N-a+1) \lambda_{a-1}
$$

If follows

$$
R_{22}=4 \frac{\lambda_{N-1}}{\lambda_{N}}=4 \sum_{i=1}^{4} x_{i}^{-2}
$$

The resulting potential is, including an oscillator potential

$$
\begin{aligned}
\frac{1}{2} W(x)= & \frac{1}{2} \omega^{2} \sum_{i=1}^{N} x_{i}^{2}+g_{1} \sum_{1 \leq i<j \leq N}\left[\left(x_{i}-x_{j}\right)^{-2}+\left(x_{i}+x_{j}\right)^{-2}\right] \\
& +g_{2} \sum_{i=1}^{N} x_{i}^{-2} \\
g_{1}= & \nu_{1}\left(\nu_{1}-1\right) \\
g_{2}= & \frac{1}{2} \nu_{2}\left(\nu_{2}-1\right)
\end{aligned}
$$

In the Sutherland case we use coordinates

$$
\begin{gathered}
\mu_{0}=\prod_{i=1}^{N} \cos ^{2} x_{i} \\
\mu_{n}(x)=\left.\mu_{0}(x) p_{n}(q)\right|_{q_{i}=\tan ^{2} x_{i}, \text { all } i} \\
n \in\{1,2, \ldots N\}
\end{gathered}
$$


From the identity

$$
\begin{aligned}
1 & =\prod_{i=1}^{N}\left(\cos ^{2} x_{i}+\sin ^{2} x_{i}\right) \\
& =\sum_{n=0}^{N} \mu_{n}(x)
\end{aligned}
$$

we learn how to eliminate $\mu_{0}$ in facour of $\left\{\mu_{n}\right\}_{n_{1}}^{N}$ so that a polynomial of $\left\{\mu_{n}\right\}_{n=0}^{N}$ remains a polynomial.

In this case the inverse Riemannian is

$$
\begin{aligned}
g_{n m}^{-1}= & 4\left\{M_{n+1, m+1}(\mu)+M_{n, m}(\mu)\right. \\
& \left.-M_{n, m+1}(\mu)-M_{n+1, m}(\mu)\right\}
\end{aligned}
$$

and the determinant decomposes as

$$
\operatorname{det} g^{-1}=4^{N}(-1)^{\left[\frac{N}{2}\right]} \mu_{0} \mu_{N} P_{1}(\mu)
$$

Now the factorization of $P_{1}(\mu)$ is

$$
P_{1}(\mu)=D_{N}^{\prime} \prod_{1 \leq i<j \leq N}\left(\cos ^{2} x_{i} \sin ^{2} x_{j}-\sin ^{2} x_{i} \cos ^{2} x_{j}\right)^{2}
$$

and we choose

$$
\begin{aligned}
& P_{2}(\mu)=\mu_{N} \\
& P_{3}(\mu)=\mu_{0}
\end{aligned}
$$

Again we have no general explicit expression for $r^{(1)}$ but

$$
\begin{aligned}
& r_{a}^{(2)}=4\left[(N-a+1) \mu_{a-1}-(N-a) \mu_{a}\right] \\
& r_{a}^{(3)}=4\left[(a+1) \mu_{a+1}-a \mu_{a}\right]
\end{aligned}
$$

so that

$$
\begin{aligned}
& R_{22}=\frac{\mu_{N-1}}{\mu_{N}}=4 \sum_{i=1}^{N} \cot ^{2} x_{i} \\
& R_{33}=\frac{\mu_{1}}{\mu_{0}}=4 \sum_{i=1}^{N} \tan ^{2} x_{i}
\end{aligned}
$$

Thus we end up with a potential

$$
\begin{aligned}
\frac{1}{2} W(x)= & g_{1} \sum_{1 \leq i<j \leq N}\left[\left(\sin \left(x_{i}-x_{j}\right)\right)^{-2}+\left(\sin \left(x_{i}+x_{j}\right)\right)^{-2}\right] \\
& +g_{2} \sum_{i=1}^{N}\left(\sin x_{i}\right)^{-2} \\
& +g_{3} \sum_{i=1}^{N}\left(\cos x_{i}\right)^{-2}
\end{aligned}
$$


where $g_{1,2}$ are as in (4.11), (4.12) and

$$
g_{3}=\frac{1}{2} \nu_{3}\left(\nu_{3}-1\right)
$$

An alternative form of the potential is obtained from

$$
\frac{g_{2}}{\sin ^{2} x}+\frac{g_{3}}{\cos ^{2} x}=\frac{g_{2}-g_{3}}{\sin ^{2} x}+\frac{4 g_{3}}{\sin ^{2} 2 x}
$$

If we set $g_{2}=g_{3}$ or $g_{3}=0$ we obtain different samples of the $B C_{N}$ or $D_{N}$ series. We mention finally that the minimal $p$-vector is in all cases

$$
\vec{p}=(1,1, \ldots 1) \in \mathbb{N}^{N}
$$

\subsection{The $F_{4}$ model}

The $F_{4}$ model belongs also to the translation noninvariant class. The Weyl group of $F_{4}$ possesses four basic polynomial invariants

$$
I_{1}(x), I_{3}(x), I_{4}(x), I_{6}(x)
$$

$\left(I_{n}\right.$ of degree $2 n$ ) which can be expressed as polynomials in the $\left\{\lambda_{n}\right\}_{n=1}^{4}$ as follows

$$
\begin{aligned}
& I_{1}=\lambda_{1} \\
& I_{3}=\lambda_{3}-\frac{1}{6} \lambda_{1} \lambda_{2} \\
& I_{4}=\lambda_{4}-\frac{1}{4} \lambda_{1} \lambda_{3}+\frac{1}{12} \lambda_{2}^{2} \\
& I_{6}=\lambda_{4} \lambda_{2}-\frac{1}{36} \lambda_{2}^{3}+\frac{1}{24} \lambda_{2}^{2} \lambda_{1}^{2}-\frac{1}{64} \lambda_{2} \lambda_{1}^{4}
\end{aligned}
$$

In these coordinates the inverse Riemannian can be given as

$$
\begin{aligned}
g_{1 m}^{-1}= & 4 m I_{m} \\
g_{33}^{-1}= & \frac{20}{3} I_{4} I_{1}-\frac{2}{3} I_{3} I_{1}^{2} \\
g_{34}^{-1}= & 8 I_{6}-3 I_{3}^{2}-\frac{13}{3} I_{4} I_{1}^{2}-\frac{3}{4} I_{3} I_{1}^{3} \\
g_{36}^{-1}= & 16 I_{4}^{2}+I_{6} I_{1}^{2}+14 I_{4} I_{3} I_{1}+\frac{5}{2} I_{3}^{2} I_{1}^{2}-\frac{1}{4} I_{4} I_{1}^{4}-\frac{5}{32} I_{3} I_{1}^{5} \\
g_{44}^{-1}= & -4 I_{4} I_{3}-2 I_{6} I_{1}+\frac{3}{4} I_{4} I_{1}^{3}+\frac{3}{4} I_{3}^{2} I_{1}+\frac{3}{16} I_{3} I_{1}^{4} \\
g_{46}^{-1}= & 8 I_{4}^{2} I_{1}+2 I_{4} I_{3} I_{1}^{2}-\frac{1}{8} I_{4} I_{1}^{5} \\
g_{66}^{-1}= & 30 I_{6} I_{4} I_{1}+\frac{21}{2} I_{6} I_{3} I_{1}^{2}-\frac{3}{32} I_{6} I_{1}^{5}+12 I_{4}^{2} I_{3}+6 I_{4} I_{3}^{2} I_{1} \\
& -\frac{3}{8} I_{4} I_{3} I_{1}^{4}+\frac{3}{4} I_{3}^{3} I_{1}^{2}+\frac{3}{1024} I_{3} I_{1}^{8}-\frac{3}{32} I_{3}^{2} I_{1}^{5}
\end{aligned}
$$


The determinant decomposes into two factors

$$
\operatorname{det} g^{-1}=\frac{1}{3072} P_{1}(I) P_{2}(I)
$$

where $P_{1}(I)$ is connected with the Vandermonde determinant squared as usual

$$
\begin{aligned}
P_{1}(I)= & -4096 I_{4}^{3}+432 I_{3}^{4}+3072 I_{6}^{2}-2304 I_{6} I_{4} I_{1}^{2} \\
& -576 I_{6} I_{3} I_{1}^{3}+864 I_{4} I_{3}^{2} I_{1}^{2}+216 I_{4} I_{3} I_{1}^{5} \\
& +432 I_{4}^{2} I_{1}^{4}+27 I_{3}^{2} I_{1}^{6}-2304 I_{6} I_{3}^{2}+216 I_{3}^{3} I_{1}^{3}
\end{aligned}
$$

or in factorized form

$$
P_{1}(I)=-16 \prod_{1 \leq i<j \leq 4}\left(x_{i}^{2}-x_{j}^{2}\right)^{2}
$$

and $P_{2}(I)$

$$
\begin{aligned}
P_{2}(I)= & 36864 I_{6}^{2}-18432 I_{6} I_{4} I_{1}^{2}-4608 I_{6} I_{3} I_{1}^{3}+32 I_{6} I_{1}^{6} \\
& -49152 I_{4}^{3}-36864 I_{4}^{2} I_{3} I_{1}+1536 I_{4}^{2} I_{1}^{4} \\
& +768 I_{4} I_{3} I_{1}^{5}-12 I_{4} I_{1}^{8}-9216 I_{4} I_{3}^{2} I_{1}^{2} \\
& -768 I_{3}^{3} I_{1}^{3}+96 I_{3}^{2} I_{1}^{6}-3 I_{3} I_{1}^{9}
\end{aligned}
$$

which factorizes as

$$
\begin{aligned}
P_{2}(I) & =-12 \lambda_{4}\left(64 \lambda_{4}-16 \lambda_{2}^{2}+8 \lambda_{2} \lambda_{1}^{2}-\lambda_{1}^{4}\right)^{2} \\
& =-12 x_{1}^{2} x_{2}^{2} x_{3}^{2} x_{4}^{2} \prod_{\nu_{2}, \nu_{3} \nu_{4} \in\{1,0\}}\left(x_{1}-\sum_{i=2}^{4}(-1)^{\nu_{i}} x_{i}\right)^{2}
\end{aligned}
$$

The $r$-vectors are

$$
\begin{array}{r}
r^{(1)}=\left(48,-2 I_{1}^{2}, 0,36 I_{4} I_{1}+12 I_{3} I_{1}^{2}-\frac{3}{16} I_{1}^{5}\right) \\
r^{(2)}=\left(48,-4 I_{1}^{2},-12 I_{3}, 24 I_{4} I_{1}+6 I_{1}^{2} I_{3}-\frac{3}{8} I_{1}^{5}\right)
\end{array}
$$

The potential resulting is

$$
\begin{gathered}
\frac{1}{2} W(x)=\frac{1}{2} \omega^{2} \sum_{1 \leq i \leq 4} x_{i}^{2}+g_{1} \sum_{1 \leq i<j \leq 4}\left[\left(x_{i}-x_{j}\right)^{-2}+\left(x_{i}+x_{j}\right)^{-2}\right] \\
+g_{2}\left\{\sum_{\substack{\nu_{2}, \nu_{3}, \nu_{4} \\
\in\{+1,0\}}} 4\left(x_{1}-\sum_{i=2}^{4} \nu_{i} x_{i}\right)^{-2}+\sum_{i=1}^{4} x_{i}^{-2}\right\} \\
\end{gathered}
$$

where $g_{1,2}$ are as in (4.11),(4.12). The minimal $p$-vector is

$$
\vec{p}=(1,2,3,5)
$$




\section{Coxeter groups, orbits and prepotentials}

The prepotentials used in the empirical constructions of sections 3 and 4 necessitate a mathematical interpretation. Let $W$ be a Coxeter group generated by the reflections

$$
\left\{s_{\alpha}\right\}
$$

where $\alpha$ are roots running over a set

$$
\Phi=\{\alpha\}_{1}^{M}
$$

The roots span an Euclidian space $V$. In this space the reflections $\left\{s_{\alpha}\right\}$ act by

$$
x \in V: s_{\alpha} x=x-2 \frac{(\alpha, x)}{(\alpha, \alpha)} \alpha
$$

If the Coxeter group $W$ is "crystallographic", it is a Weyl group (for more details see [9]).

We denote a set of basic polynomial invariants of $W$ by

$$
\left\{z_{1}(x), \ldots, z_{n}(x)\right\}, \quad n=\operatorname{dim} V
$$

Invariance means

$$
\begin{aligned}
z_{i}\left(w^{-1} x\right) & =z_{i}(x) \\
& =w z_{i}(x)
\end{aligned}
$$

for all $w \in W$. The Jacobian for the transition $\left\{x_{j}\right\} \rightarrow\left\{z_{i}\right\}$

$$
J=\operatorname{det}\left\{\frac{\partial z_{i}}{\partial x_{j}}\right\}
$$

can be factorized as follows ([9], Proposition 3.13).

Each reflection $s_{\alpha}$ leaves a hyperplane $H_{\alpha}$ in $V$ pointwise fixed, let $H_{\alpha}$ be given by a linear function $l_{\alpha}$

$$
l_{\alpha}(x)=0
$$

Then due to the proposition

$$
J=C \prod_{\alpha \in \Phi^{+}} l_{\alpha}(x)
$$

with $\Phi^{+}$the set of positive roots. The proof of this proposition is rather elementary.

For any inverse Riemann tensor $\left\{g^{-1}\right\}$ of Sections 3 and 4 we obtain this way

$$
\operatorname{det} g_{a b}^{-1}=C^{2} \prod_{\alpha \in \Phi^{+}} l_{\alpha}(x)^{2}
$$


If $\Phi$ decomposes into orbits under $W$

$$
\Phi=\bigcup_{i} \Phi_{i}
$$

then

$$
P_{i}=\prod_{\alpha \in \Phi_{i}^{+}} l_{\alpha}(x)^{2}
$$

is an invariant polynomial under action of $W$ and therefore a polynomial in the basic invariants

$$
P_{i}=P_{i}\left(z_{1}, \ldots, z_{n}\right)
$$

These polynomials are the prepotentials constructed in Sections 3 and 4 . The factorization of these prepotentials as quoted at the end of Section 2 (eqns. (2.35),(2.36) ) and used throughout in Sections 3 and 4 is based on (5.11).

We emphasize that our empirical results of Sections 3 and 4 indicate the validity of further mathematical propositions which could not be traced in the literature:

1. an analogous factorization theorem for the trigonometric invariants;

2. the polynomial properties ("integrability") of the functions $r^{(i)}(z)(2.22)$.

Now we return to the $A G_{3}$ model of Section 3. We identify the roots involved in a model using $(5.7),(5.9)$

$$
\begin{aligned}
l_{\alpha}(x) & =\left(\alpha^{\vee}, x\right) \\
\left(\alpha^{\vee}\right. & \left.=\frac{2 \alpha}{(\alpha, \alpha)}, \text { the "dual" of } \alpha\right)
\end{aligned}
$$

and the Sutherland version whose potential is

$$
\frac{1}{2} W(x)=\sum_{\text {orbits } i} g_{i} \sum_{\alpha \in \Phi_{i}^{+}}\left[\sin l_{\alpha}(x)\right]^{-2}
$$

Thus the simple roots of $A_{3}$

$$
\begin{aligned}
\alpha_{1} & =e_{1}-e_{2} \\
\alpha_{2} & =e_{2}-e_{3} \\
\alpha_{3} & =e_{3}-e_{4}
\end{aligned}
$$

are completed by a fourth root in $A G_{3}$

$$
\alpha_{4}=e_{3}+e_{4}-e_{1}-e_{2}
$$

The corresponding Coxeter-diagram is shown in Fig. 1. It belongs to the 


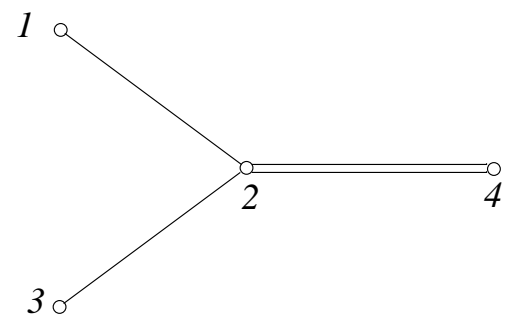

Figure 1: Coxeter diagram of $\hat{B}_{3}$

affine Coxeter group $\hat{B}_{3}$ ([9], Figure 1 in Section 2.4).

The coordinates of the $\hat{B}_{3}$ root space with respect to the standard basis $\left\{f_{i}\right\}_{i=1}^{3}$ are denoted $\left\{\xi_{i}\right\}_{i=1}^{3}$, those of $A G_{3}$ with respect to the standard basis $\left\{e_{i}\right\}_{i=1}^{4}$ by $\left\{x_{i}\right\}_{i=1}^{4}$ as before. The simple roots of $B_{3}$ are

$$
\beta_{1}=f_{1}-f_{2}, \quad \beta_{1}=f_{2}-f_{3}, \quad \beta_{3}=f_{3}
$$

and $\hat{B}_{3}$ is obtained by adjoining

$$
\beta_{4}=-f_{1}-f_{2}
$$

It follows that

$$
s_{4}\left(\begin{array}{l}
\xi_{1} \\
\xi_{2} \\
\xi_{3}
\end{array}\right)=\left(\begin{array}{c}
-\xi_{2} \\
-\xi_{1} \\
\xi_{3}
\end{array}\right)
$$

leaves the Coxeter invariants of $B_{3}$

$$
\begin{aligned}
& \lambda_{1}(\xi)=\sum_{1 \leq i \leq 3} \xi_{i}^{2} \\
& \lambda_{2}(\xi)=\sum_{1 \leq i<j \leq 3} \xi_{i}^{2} \xi_{j}^{2} \\
& \lambda_{3}(\xi)=\xi_{1}^{2} \xi_{2}^{2} \xi_{3}^{2}
\end{aligned}
$$

invariant, too. This suggests the equivalence of the $A G_{3}$ and the $B_{3}$ models.

An explicit identification of the simple roots

$$
\begin{aligned}
& f_{1}=\frac{1}{2}\left(e_{1}-e_{2}-e_{3}+e_{4}\right) \\
& f_{2}=\frac{1}{2}\left(-e_{1}+e_{2}-e_{3}+e_{4}\right) \\
& f_{3}=\frac{1}{2}\left(-e_{1}-e_{2}+e_{3}+e_{4}\right)
\end{aligned}
$$

gives $(i, j \in\{1,2,3\})$

$$
\begin{aligned}
x_{i}-x_{j} & =\xi_{i}-\xi_{j} \\
x_{4}-x_{j} & =\sum_{i(\neq j)} \xi_{i}
\end{aligned}
$$




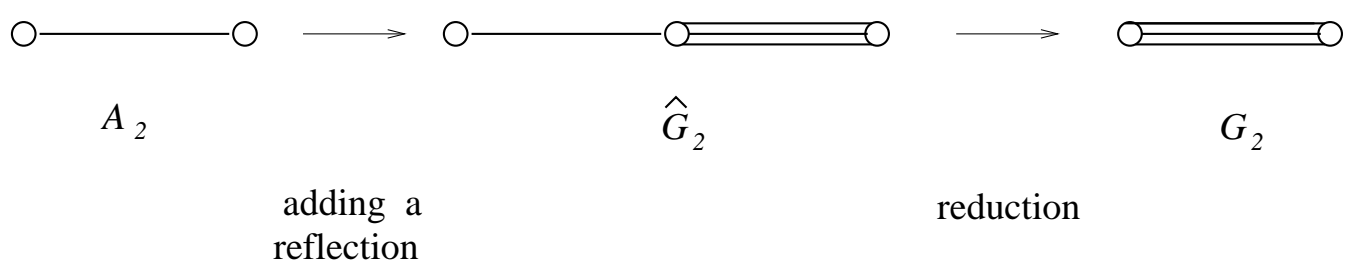

Figure 2: Extending the Coxeter diagram of $A_{2}$ to $\hat{G}_{2}$ and reduction to $G_{2}$

It follows

$$
\begin{aligned}
& g_{1} \sum_{1 \leq i<j \leq 4}\left[\sin \left(x_{i}-x_{j}\right)\right]^{-2}+\frac{1}{4} g_{2} \sum_{3 \text { cases }}\left[\sin \frac{1}{2}\left(x_{i}+x_{j}-x_{k}-x_{l}\right)\right]^{-2} \\
= & g_{1} \sum_{1 \leq i<j \leq 3}\left\{\left[\sin \left(\xi_{i}-\xi_{j}\right)\right]^{-2}+\left[\sin \left(\xi_{i}+\xi_{j}\right)\right]^{-2}\right\}+\frac{1}{4} g_{2} \sum_{i=1}^{3}\left[\sin \xi_{i}\right]^{-2}
\end{aligned}
$$

Moreover the rational invariants (3.64) can be identified with the invariants (5.20)- $-(5.22)$

$$
\begin{aligned}
\mu_{2}(x) & =-\frac{1}{2} \lambda_{1}(\xi) \\
\mu_{3}(x) & =+\frac{1}{4} \lambda_{3}(\xi) \\
\mu_{4}(x) & =-\frac{1}{4} \lambda_{2}(\xi)+\frac{1}{16} \lambda_{1}(\xi)^{2}
\end{aligned}
$$

This establishes the equivalence between the two models.

Our method involves a reduction of the affine Coxeter group $\hat{B}_{3}$ to the Coxeter group $B_{3}$ having the same invariants. It may therefore be of interest that the construction performed in 四 is analogous (see Fig. 2). 


\section{References}

[1] M.A: Olshanetsky and A.M. Perelomov, Lett. Math. Phys. 2 (1977) 7-13, and Phys. Reports 94 (1983) 313.

[2] A.V. Turbiner, "Lie algebras and linear operators with invariant subspace", in "Lie algebras, cohomologies and new findings in quantum mechanics" (N. Kamran and P.J. Olver eds.) AMS, Vol 160, pp. 263310, 1994;

"Lie-algebras and quasi-exactly-solvable differential equations", Vol 3: New Trends in Theoretical Developments and Computational Methods, Chapter 12, CRC Press (N. Ibragimov ed.) pp. 331-366, 1995 (hep-th $9409068)$.

[3] W. Rühl and A. Turbiner, Mod. Phys. Letters A10 (1995) 2213 (hep-th 9506105).

[4] M. Rosenbaum, A. Turbiner, A. Capella, Solvability of the $G_{2}$ Integrable System; (sol-int 9707005).

[5] L. Brink, A. Turbiner, N. Wyllard, J. Math. Phys. 39 (1998) 1285.

[6] O. Haschke, W. Rühl, Exactly solvable dynamical systems in the neighborhood of the Calogero model, to appear (hep-th 9803169); in eq. (7.14) $a_{7}$ should read $a_{7}-3$.

[7] J. Wolfes, Ann. Phys. (N.Y.) 85 (1974) 454.

[8] O. Haschke, W. Rühl, Construction of exactly solvable quantum models of Calogero and Sutherland type with translation invariant four-particle interactions, (hep-th 9807194)

[9] J.E. Humphreys, Reflection groups and Coxeter groups, Cambridge University Press 1990 